\title{
ARTICLE
}

Clinical Study

\section{First results from five multidisciplinary diagnostic centre (MDC) projects for non-specific but concerning symptoms, possibly indicative of cancer}

\author{
D. Chapman $\mathbb{D}^{1}$, V. Poirier $\mathbb{D}^{1}$, D. Vulkan $\mathbb{D}^{2}$, K. Fitzgerald $\mathbb{D}^{1}$, G. Rubin $\mathbb{D}^{3}$, W. Hamilton $\mathbb{D}^{4}$ and \\ S. W. Duffy $\mathbb{D}^{2}$ on behalf of the ACE MDC projects
}

\begin{abstract}
BACKGROUND: Patients with non-specific symptoms often experience longer times to diagnosis and poorer clinical outcomes than those with site-specific symptoms. This paper reports initial results from five multidisciplinary diagnostic centre (MDC) projects in England, piloting rapid referral for patients with non-specific symptoms.

METHODS: The evaluation covered MDC activity from 1st December 2016 to 31st July 2018, with projects using a common dataset. Logistical regression analyses were conducted, with a diagnosis of any cancer as the dependent variable. Exploratory analysis was conducted on presenting symptoms and diagnoses of cancer, and on comparisons within these groupings.

RESULTS: In total, 2961 patients were referred into the MDCs and 241 cancers were diagnosed. The pathway detected cancers across a broad range of tumour sites, including several rare and less common cancers. An association between patient age and cancer was identified $(p<0.001)$. GP 'clinical suspicion' was identified as a strong predictor of cancer $(p=0.006)$, with a reduced association with cancer observed in patients with higher numbers of GP consultation before referral $(p=0.008)$.

CONCLUSIONS: The MDC model diagnoses cancer in patients with non-specific symptoms, with a conversion rate of $8 \%$, demonstrating the diagnostic potential of a non-site-specific symptomatic referral pathway.
\end{abstract}

British Journal of Cancer (2020) 123:722-729; https://doi.org/10.1038/s41416-020-0947-y

\section{BACKGROUND}

Patients presenting with non-specific but concerning symptoms (hereafter 'non-specific symptoms') account for a significant proportion of cancer diagnoses in England. ${ }^{1}$ Some of these symptoms, such as unexplained weight loss, non-specific abdominal pain, fatigue and nausea/vomiting, have a low predictive value for individual cancers. ${ }^{2-4}$ However, whilst the risk of specific cancers may be low, the overall risk of harbouring cancer of any type is higher, ${ }^{2,5}$ suggesting that swift investigation for patients with non-specific symptoms is merited. In the absence of an established pathway to ensure a timely and planned referral, these patients have often experienced longer times to diagnosis than those presenting with recognised high-risk symptoms indicative of specific cancers. ${ }^{4,6,7}$

As non-specific symptoms can be caused by a range of conditions, of which cancer is only one, identifying the appropriate diagnostic test and referral route can be challenging. Consequently, patients with non-specific symptoms more frequently have multiple GP consultations before referral, ${ }^{6-8}$ potentially contributing to longer intervals from presentation to diagnosis of cancer. ${ }^{6,7}$ Patients in this cohort are also associated with higher rates of cancer diagnosis via emergency presentation ${ }^{6,9}$ and of late-stage cancer diagnoses, ${ }^{6}$ both
\end{abstract}

of which contribute to poorer clinical outcomes ${ }^{10-12}$ and poorer patient experience of care. ${ }^{13}$

The multidisciplinary diagnostic centre (MDC) concept aims to improve outcomes for patients presenting with non-specific symptoms by providing rapid access to a range of diagnostic tests within a single diagnostic pathway, with a number of specialists working together to speed up diagnosis for the patient. ${ }^{11}$ The MDC concept was first trialled nationally in Denmark in 2012 as part of its three-legged cancer strategy, ${ }^{14-16}$ with Diagnostic Centres established alongside arrangements for the investigation of low-risk but not no-risk symptoms (Yes/No Clinic), and an urgent referral pathway for specific alarm symptoms. The Danish pathway comprises a filter function conducted by the GP, which includes a range of pre-specified diagnostic tests, followed by referral into the Diagnostic Centre itself if serious symptoms persist without a diagnosis being reached. ${ }^{14}$

The Accelerate Coordinate Evaluate (ACE) Programme ${ }^{17}$ of interventions is aimed at improving the pathway to cancer diagnosis, and thereby improving cancer outcomes, through the provision of evidence-based information and support. Wave 2 of the programme involved the establishment of a cluster of five projects in England to examine and evaluate the concept of

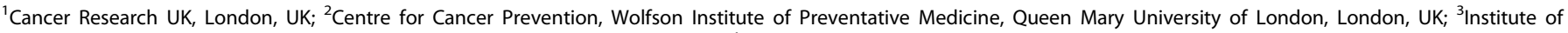
Population Health Sciences, Newcastle University, Newcastle upon Tyne, UK and ${ }^{4}$ University of Exeter Medical School (Primary Care), Devon, UK

Correspondence: D Chapman (dave.chapman@cancer.org.uk)

Members of the ACE MDC projects are listed above Acknowledgements.
}

Received: 1 August 2019 Revised: 19 May 2020 Accepted: 4 June 2020

Published online: 6 July 2020 
Table 1. Initial MDC arrangements by individual project.

Airedale

Launch date 17 th January 17

Referral criteria Persistent unexplained abdominal pain, persistent unexplained weight loss, non-specific but concerning symptoms with a high risk of cancer, GP clinical suspicion and too unwell for 2-Week Wait referral

Referral route GP, A\&E and Secondary Care Clinic

Greater Manchester

Launch date 3rd Mar 17 (Royal Oldham Hospital), 13th December 16 (Wythenshawe Hospital)

Referral criteria Non-specific abdominal pain, unexplained weight loss, severe unexplained fatigue, nausea/appetite loss, lymphadenopathy, hepatomegaly, splenomegaly, bloating, GP clinical suspicion and non-iron- deficiency anaemia

Referral route GP

Leeds

Launch date 31st January 17

Referral criteria Appetite loss + nausea (unexplained, 40 and over), weight loss (unexplained, 40 and over), abdominal pain without rectal bleeding or weight loss (<3-month duration or recent change in character/severity, 50 and over), anaemia (non-iron deficiency, without evidence of bleeding, 50 years and over), hypercalcaemia (unexplained and persisting $<12$ months), thrombocythemia (unexplained and persisting $<12$ months and GP clinical suspicion and general condition ("poor" general condition)

Referral route GP, Acute Medicine

London

Launch date 1st May 17 (North Middlesex), 1st April 17 (UCLH), 1st December 16 (Queens-BHRUT), 1st January 18 (Royal Free Hospital) and 15 th September 2017 (Southend)

Referral criteria Broad range of abdominal symptoms with no clear referral pathway and where patients cannot wait for routine referral, including new unexplained abdominal pain, unexplained weight loss, persistent nausea/appetite loss, GP clinical suspicion and painless jaundice

Referral route GP

Oxford

Launch date 15th March 17

Referral criteria Severe unexplained fatigue, unexplained weight loss, persistent nausea or appetite loss, new atypical pain, unexplained laboratory findings, no organ-specific symptoms, no symptoms fulfilling referral via the standard 2-week wait pathway, over 40 years old and GP clinical suspicion ("gut feeling")

Referral route GP

MDC-based pathways for patients presenting with non-specific symptoms.

In this study, we evaluate the early outcomes for the ACE Programme's pilot MDC sites, in respect to patient characteristics, cancer diagnoses and stage at diagnosis, and associations between patient factors and diagnosis of cancer.

\section{METHODS}

Project structure

ACE MDC Programme activity was based within five projects in England, with ten operational MDC pilot sites (Airedale, Greater Manchester $(\times 2)$, Leeds, London $(\times 5)$ and Oxford). All projects focused on the development of a pilot pathway for patients with non-specific symptoms and, whilst projects configured their approaches to reflect local healthcare systems and clinical priorities, a core set of distinguishing features were evident across all pilot sites. ${ }^{18}$

As the programme was structured around a service evaluation of pilot pathways, a level of heterogeneity was introduced amongst the projects to test differing MDC approaches and to assess pathway adaptability. However, regarding the clinical interpretation of the patient's presenting symptoms, all five projects adhered to two fundamental principles for referral:

1. That the patient must be considered as being of clinical concern, with non-specific symptoms potentially indicative of cancer (or other serious disease) and

2. That their presenting symptoms are not sufficiently clear to indicate an appropriate tumour-specific urgent referral pathway.
As the MDC model focused on the diagnosis of any cancer presenting with non-specific symptoms, and not being limited to new cancer cases only, the presence of a previous cancer was permitted, provided that the patient met the pathway's two governing principles for referral. Despite being considered a sitespecific symptom, painless jaundice was included as a referral criterion in London MDC to reflect locally determined clinical priorities.

Table 1 provides details of the date at which the intervention was implemented, and the referral routes and the criteria for each MDC project.

Research methods

A programme dataset was agreed across the projects to ensure a robust evaluation. It included data items based on the English cancer outcomes and services dataset, ${ }^{19}$ and additional projectspecific items focusing on secondary care presentation, diagnostic process of cancers and other diseases. Data items were collected locally from a combination of primary care referral forms and secondary care data systems.

Data management arrangements varied between MDC projects; a combination of local healthcare IT systems and stand-alone systems were used, which impacted upon the availability of certain data, for example, missing performance status and comorbidity data items. The data items were captured in near real time where possible either by automated data extraction or entry by clinical staff. Some additional data required manual entry at a later date.

All projects had a nominated data lead, and project data items were submitted to programme evaluators on a quarterly basis, 
724

where some recoding rules were applied to align the data for analysis.

A list of thirteen symptoms (plus 'other') was included in the agreed dataset. In subsequent analyses, these thirteen symptoms are referred to together as 'selected symptoms'. The range of selected symptoms was developed with clinical guidance and chosen to describe a general pattern of seriously unwell patients, whose presentation is suggestive of cancer, but does not indicate a specific diagnostic approach. In some instances, this includes conditions and signs that are not strictly symptoms. In addition to the thirteen 'selected symptoms', other symptoms were recorded as part of open-ended data recording; these have been classified as 'non-selected' symptoms.

Patients presented with non-specific symptoms in either isolation or in varying combinations. Clinical judgement formed part of the decision to refer, and all relevant factors were considered as part of this process and captured on referral templates where relevant. Eligible referral criteria were included on MDC referral templates to aid consistency and quality, and to clearly indicate patient symptoms as appropriate.

As indicated in Table 1, individual MDC hospital sites became operational at different times and began contributing data at different points during the evaluation period, from December 2016 to January 2018, with this evaluation covering MDC activity up to 31 st July 2018.

As projects have uniformly applied their selected referral criteria, filter tests and diagnostic approaches to their own pilot sites, data in this study are aggregated to a project and programme level to provide greater scope for analysis. Although measures were taken centrally to support data consistency and completeness, a certain degree of variation in data interpretation and formatting was created at MDC level. Additional variations in the data completeness across the pilot sites were observed, often due to manpower issues. Certain data items have approximately one-third of data classified as 'unknown'. Performance status ${ }^{20}$ was not recorded for 897 patients (30\% of the total), and comorbidity $^{21}$ information was absent for 729 (25\%). Performance status and comorbidity, both of which had relevant data recorded for four MDC projects only, were retained for the purpose of describing the characteristics of the overall cohort.

The number of primary care consultations was missing for 1351 patients (46\%), and symptom duration for 1012 (34\%). In each case, the extent of missing data varied by project site. Sensitivity analyses have been conducted on primary care consultation history and symptom duration to assess the likely impact of missing data, as these data items have been included in logistic regression analyses. Missing data are noted as unknown in the tables, and records with missing data have been excluded from all analyses relating to those fields (i.e. blanks have been omitted from analyses).

\section{Statistical analysis}

Patients were deemed to have a cancer if their diagnostic outcome was recorded as either "New cancer" or "Recurrence".

A series of single-variable logistic regressions were carried out, with diagnosis of any cancer as the dependent variable. Independent variables considered were gender, age, number of primary care consultations prior to referral (up to three, or three or more), duration of symptoms (five categories), the presence of each of the thirteen selected symptoms, including one general symptom (the classification of 'pain' as a collective grouping of all pain-associated symptoms, $85 \%$ of cases being abdominal pain) and the number of selected symptoms reported.

To assess the likely impact of missing data relating to previous primary care consultations and reported duration of symptoms, two approaches were undertaken. The first approach was to omit those sites where, for each variable considered, no data were recorded for $20 \%$ or more of patients. The second approach was to replace all missing values with, in turn, the highest or lowest possible values of the variable, therefore creating a 'best case' and 'worst case' scenario for each variable. The results carried out using these approaches did not provide strong evidence to reconsider the conclusions reached. The results are presented in Supplementary Information A.

Further logistic regression was carried out, taking multiple independent variables into account at the same time. Statistically significant (at the 0.05 level) factors identified in the single-variable analyses (gender, age, fewer than 3 previous primary care consultations, total number of selected symptoms, GP 'clinical suspicion', nausea/appetite loss, fatigue and anaemia) were included in multiple regression to assess possible confounding effects. Multivariable analyses are presented as 'adjusted' rates alongside the relevant 'unadjusted' single-variable rates.

\section{RESULTS}

There were 2961 patients referred to the pilot MDCs. Table 2 shows the demographic characteristics, comorbidity score and primary care consultation history of the patients referred. Patients' ages ranged from 17 to 97 years, with an average of 66.7 (SD 14.9). Of the patients, $44 \%$ were male, $40 \%$ had some degree of physical impairment (based on recorded performance status of 2064 completed patient records) and $27 \%$ had moderate or severe comorbidities (based on 2232 completed patient records). Of those with known primary care consultation history, $28 \%$ had 3 or more previous consultations.

Table 3 shows the frequency of selected symptoms of referred patients. The most common symptom was weight loss $(66 \%$ of those for whom symptoms were recorded) followed by GP 'clinical suspicion' (36\%) based on the GP's clinical judgement regarding overall suspicion of malignancy ${ }^{2}$ and pain (32\%). In total, $61 \%$ of patients presented with more than one of the thirteen selected symptoms (decreasing to $57 \%$ when including non-selected symptoms, but excluding GP 'clinical suspicion' and family concern). Of those with symptom duration data, $55 \%$ reported a symptom duration of 3 months or more.

Of the patients referred, $240(8 \%)$ were diagnosed with cancer, 239 with a single cancer and one patient diagnosed with two cancers (one urological and one lower $\mathrm{Gl}$ ). In the 16 cases where cancer was classified as 'recurrence', all patients presented without any recognised alarm symptoms. In addition to diagnoses of cancer, over $50 \%$ of cases within the MDC were diagnosed with non-cancer conditions; of those with a given diagnosis, $40 \%$ were related to diseases of the digestive system.

Table 4 shows the sites of the cancers diagnosed. The commonest were upper $\mathrm{Gl}$ (including 25 cases of pancreatic cancer) and lung, with 53 and 52 cancers diagnosed, respectively.

Table 5 shows the results of logistic regression for associations of selected factors with a diagnosis of any cancer. When considered in isolation, several factors displayed strong associations $(p<0.05)$ with a diagnosis of cancer. However, when accounting for all significant individual variables at the same time, strong associations were only identified between a cancer diagnosis and increasing age $(p<0.001)$, and with GP 'clinical suspicion' $(p=0.006)$ as a predictor of cancer. In addition, an association was maintained between patients with more than three GP consultations prior to referral $(p=0.008)$ and a reduced likelihood of a cancer diagnosis.

The remaining factors, when included in multiple regression, no longer recorded a statistically significant association with cancer diagnosis, namely, gender $(p=0.172)$, nausea/appetite loss ( $p=$ $0.51)$, fatigue $(p=0.36)$, anaemia $(p=0.64)$ and the number of symptoms reported $(p=0.53)$.

Table 6 shows the stage distribution of cancers diagnosed. For all cancers, the majority were at stage III or IV. There were, however, notable proportions at stage I and II for upper GI, lung 
First results from five multidisciplinary diagnostic centre (MDC)...

Table 2. Characteristics of the patients referred to the MDC.

\begin{tabular}{|c|c|c|}
\hline Total number of patients & 2961 & \\
\hline Sex & $N$ & $\%$ \\
\hline Male & 1304 & 44.2 \\
\hline Female & 1646 & 55.8 \\
\hline Unknown & 11 & $\mathrm{~N} / \mathrm{A}$ \\
\hline \multicolumn{3}{|l|}{ Age group } \\
\hline Under 30 & 52 & 1.8 \\
\hline $30-39$ & 109 & 3.7 \\
\hline $40-49$ & 231 & 7.8 \\
\hline $50-59$ & 480 & 16.2 \\
\hline $60-69$ & 649 & 21.9 \\
\hline 70-79 & 814 & 27.5 \\
\hline $80-89$ & 567 & 19.2 \\
\hline $90-99$ & 59 & 2 \\
\hline Mean (SD) & $66.7(14.9)$ & \\
\hline Median & 69 & \\
\hline \multicolumn{3}{|l|}{ Performance status } \\
\hline 0 & 1239 & 60.0 \\
\hline 1 & 462 & 22.4 \\
\hline 2 & 232 & 11.2 \\
\hline 3 & 113 & 5.5 \\
\hline 4 & 18 & 0.9 \\
\hline Unknown & 897 & $\mathrm{~N} / \mathrm{A}$ \\
\hline \multicolumn{3}{|l|}{ Comorbidity score } \\
\hline 0 & 699 & 31.3 \\
\hline 1 & 938 & 42.0 \\
\hline 2 or $2 / 3$ & 406 & 18.2 \\
\hline 3 & 189 & 8.5 \\
\hline Unknown & 729 & $\mathrm{~N} / \mathrm{A}$ \\
\hline \multicolumn{3}{|l|}{ Primary care consultations } \\
\hline None & 114 & 7.1 \\
\hline 1 & 539 & 33.5 \\
\hline 2 & 501 & 31.1 \\
\hline 3 & 221 & 13.7 \\
\hline 4 & 91 & 5.7 \\
\hline 5 & 50 & 3.1 \\
\hline More than 5 & 94 & 5.8 \\
\hline Unknown & 1351 & $\mathrm{~N} / \mathrm{A}$ \\
\hline
\end{tabular}

and haematological cancers. For the haematological cancers, however, stage was unavailable for more than $50 \%$ of the cancers.

\section{DISCUSSION}

The most important aspect of the study is that $8 \%$ of referrals resulted in a cancer diagnosis, with 241 cancers diagnosed from a referral cohort of 2961 patients. Thus, this study of activity within the ACE pilot sites has shown that MDC-based pathways diagnose cancer in patients presenting with non-specific symptoms. Although most referrals do not result in a cancer diagnosis for the patient, the reported conversion rate of $8 \%$ exceeds the positive predictive value of $3 \%$ recommended for urgent definitive investigation, ${ }^{2}$ and remains consistent with national guidance.

The MDCs diagnosed cancers across a broad range of tumour sites, which is to be expected given the purpose of the pathway and the non-specific symptoms of patients. Encouragingly, several of these diagnoses were of rare and less common cancers,
Table 3. Nature, number and duration of symptoms in patients referred to the MDC.

\begin{tabular}{|c|c|c|c|c|c|c|}
\hline \multirow[t]{2}{*}{ Reported symptoms } & \multicolumn{2}{|c|}{$\begin{array}{l}\text { As only } \\
\text { selected } \\
\text { symptom }\end{array}$} & \multicolumn{2}{|c|}{$\begin{array}{l}\text { With other } \\
\text { symptoms }\end{array}$} & \multicolumn{2}{|c|}{$\begin{array}{l}\text { All } \\
\text { occurrences }\end{array}$} \\
\hline & $N$ & $\%$ & $N$ & $\%$ & $N$ & $\%$ \\
\hline Weight loss & 579 & 20.3 & 1,295 & 45.4 & 1874 & 65.7 \\
\hline GP clinical suspicion & 124 & 4.3 & 904 & 31.7 & 1028 & 36.1 \\
\hline Pain & 248 & 8.7 & 678 & 23.8 & 926 & 32.5 \\
\hline Nausea/appetite loss & 26 & 0.9 & 817 & 28.7 & 843 & 29.6 \\
\hline Fatigue & 18 & 0.6 & 528 & 18.5 & 546 & 19.2 \\
\hline Anaemia & 40 & 1.4 & 256 & 9 & 296 & 10.4 \\
\hline Change in bowel habit & 9 & 0.3 & 119 & 4.2 & 128 & 4.5 \\
\hline General condition & 6 & 0.2 & 97 & 3.4 & 103 & 3.6 \\
\hline Respiratory problem & 5 & 0.2 & 67 & 2.4 & 72 & 2.5 \\
\hline Thrombocytosis & 4 & 0.1 & 19 & 0.7 & 23 & 0.8 \\
\hline Jaundice & 9 & 0.3 & 10 & 0.4 & 19 & 0.7 \\
\hline Hypercalcaemia & 0 & 0 & 8 & 0.3 & 8 & 0.3 \\
\hline New-onset diabetes & 0 & 0 & 3 & 0.1 & 3 & 0.1 \\
\hline Other symptom(s) (only) & 43 & 1.5 & $\mathrm{~N} / \mathrm{A}$ & N/A & 43 & 1.5 \\
\hline \multicolumn{5}{|l|}{ Unknown } & 110 & $\mathrm{~N} / \mathrm{A}$ \\
\hline \multicolumn{5}{|c|}{ Number of selected symptoms reported } & $N$ & $\%$ \\
\hline \multicolumn{5}{|l|}{ None } & 43 & 1.5 \\
\hline \multicolumn{5}{|l|}{1} & 1068 & 37.5 \\
\hline \multicolumn{5}{|l|}{2} & 879 & 30.8 \\
\hline \multicolumn{5}{|l|}{3} & 515 & 18.1 \\
\hline \multicolumn{5}{|l|}{4} & 244 & 8.6 \\
\hline \multicolumn{5}{|l|}{5} & 90 & 3.2 \\
\hline \multicolumn{5}{|l|}{6} & 12 & 0.4 \\
\hline \multicolumn{7}{|c|}{ Overall number of symptoms per case $e^{* *}$} \\
\hline \multicolumn{3}{|l|}{1 or fewer } & & & 1234 & 43.3 \\
\hline \multicolumn{3}{|l|}{2 or more } & & & 1617 & 56.7 \\
\hline \multicolumn{7}{|l|}{ Reported symptom duration } \\
\hline \multicolumn{3}{|l|}{ Less than 1 week } & & & 25 & 1.3 \\
\hline \multicolumn{3}{|l|}{$1-4$ weeks } & & & 348 & 17.9 \\
\hline \multicolumn{3}{|l|}{ 5-12 weeks } & & & 507 & 26.0 \\
\hline \multicolumn{3}{|l|}{ 3-6 months } & & & 573 & 29.4 \\
\hline \multicolumn{3}{|l|}{ Over 6 months } & & & 496 & 25.5 \\
\hline \multicolumn{3}{|l|}{ Unknown } & & & 1012 & $\mathrm{~N} / \mathrm{A}$ \\
\hline
\end{tabular}

Table 4. Anatomical sites of the 240 patients diagnosed with cancer.

\begin{tabular}{lcr}
\hline Cancer site & Number & $\%$ \\
\hline Upper GI & 53 & 22 \\
Lung & 52 & 22 \\
Haematological & 31 & 13 \\
Lower Gl & 30 & 13 \\
Urological & 30 & 13 \\
Breast & 15 & 6 \\
Sarcoma & 6 & 3 \\
Lower Gl and urological & 1 & 0 \\
Other & 20 & 8 \\
Unknown & 2 & 1 \\
\hline
\end{tabular}


Table 5. Logistic regression results for associations of selected factors with diagnosis of cancer.

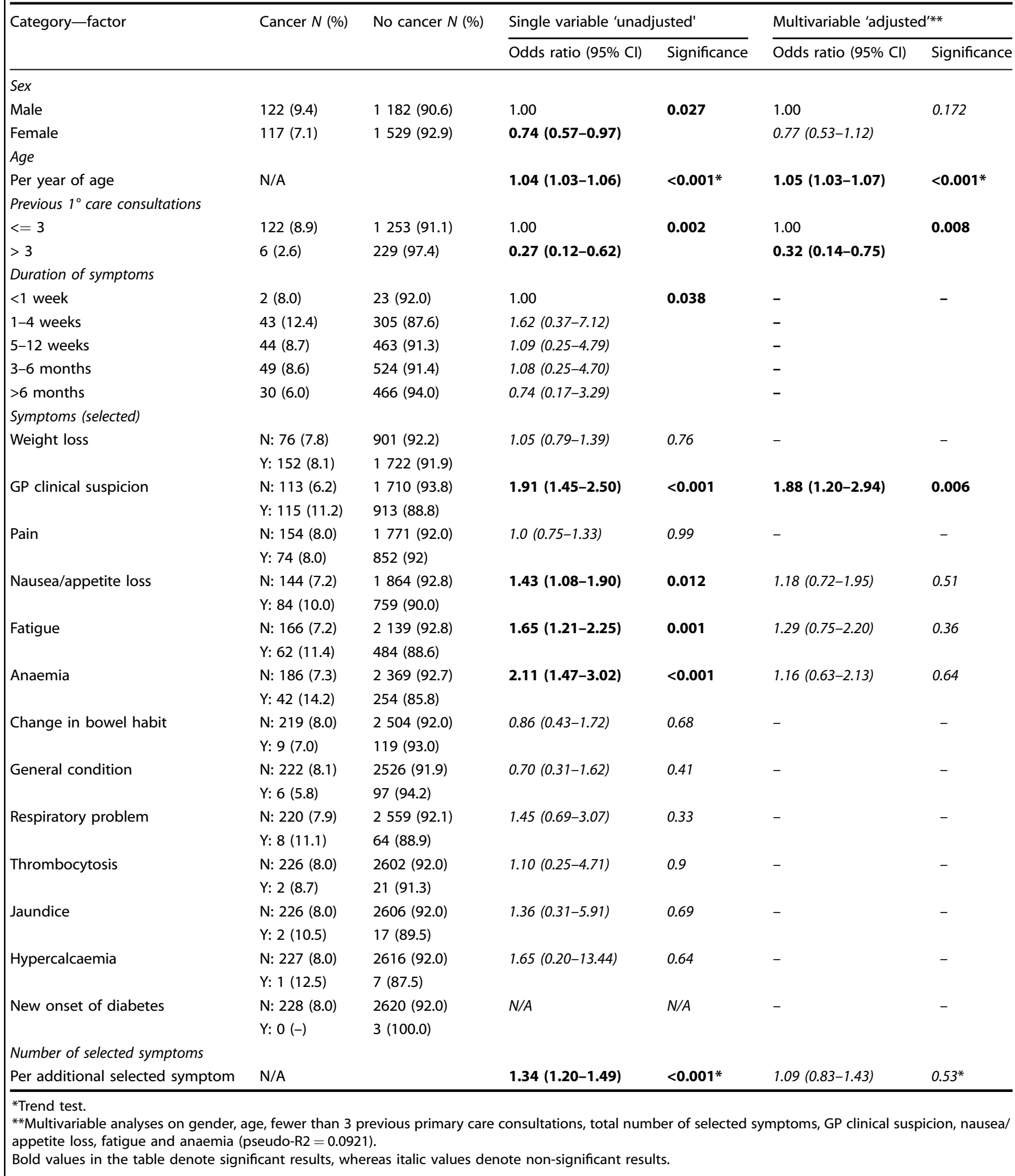

reflecting the higher risk of harbouring cancer of any type amongst this patient group. ${ }^{2,5}$

Patient age was found to be the most important factor associated with a cancer diagnosis $(p<0.001)$ and, once age was controlled for, the significance of other factors was reduced. A significant relationship was also detected between a diagnosis of cancer and GP 'clinical suspicion' $(p=0.006)$, and is consistent with GP 'clinical suspicion' being a powerful predictor of cancer. ${ }^{22}$ The reduced association between a cancer diagnosis and patients with more than three GP consultations prior to referral is also 
Table 6. Stage distributions of cancers diagnosed.

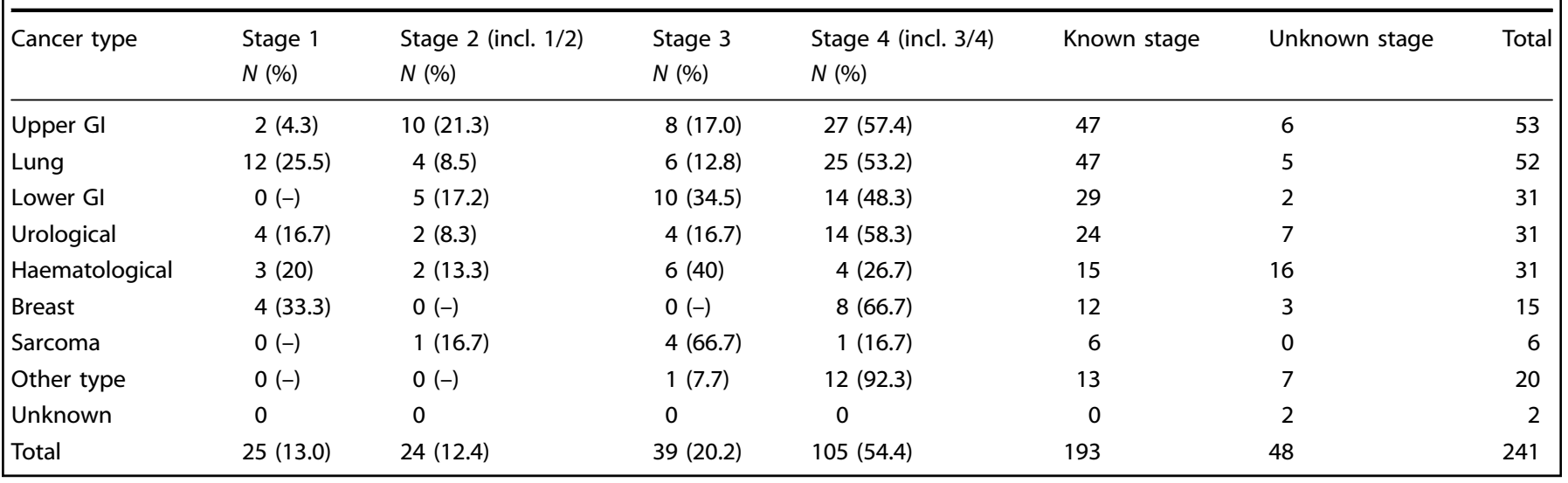

noteworthy, despite partial data completeness being observed at a project level. Within the context of this study, it may be that where they have a strong suspicion of cancer, GPs are more likely to pursue available options such as the MDC to achieve earlier referral for these patients. Consequently, many of the cases of cancer in this study may have been suspected and referred by the GP during the first few consultations. Analyses of a similar patient cohort suggest that, without a rapid referral route such as the MDC, cancer patients with non-specific symptoms often experienced greater numbers of GP consultations before referral than those with site-specific symptoms, ${ }^{6}$ thus highlighting the potential for this new approach.

The study has provided information that may be useful in describing this new referral cohort. Notable proportions of patient referrals in four MDC projects reported poor performance and comorbidity. A strong association between these factors and increasing age obfuscates the nature and significance of these characteristics, ${ }^{23,24}$ but is useful in illustrating the complexity of diagnostic decision-making for this patient group.

Several presenting symptoms recorded increased odds of a cancer diagnosis, most notably fatigue (OR 1.29 (0.75-2.20)), nausea/appetite loss (OR $1.18(0.72-1.95)$ ) and anaemia (OR 1.16 (0.63-2.13)), whilst the risk of cancer also marginally increased per additional symptom reported (OR 1.09 (0.83-1.43)), although none of these were statistically significant. There was no clear effect of the reported duration of symptoms overall, but data suggested an increased association for a duration of 1-4 weeks, which then declined for longer durations. However, this may be influenced by challenges regarding objective and accurate symptom recall, ${ }^{3}$ and a higher degree of precision in remembering the onset of more recent symptoms. ${ }^{25}$ Collectively, such information may be helpful in describing the type of patient and presenting a profile that may benefit from urgent referral for non-specific symptoms.

There were relatively large proportions of subjects with stage IV cancer. As several of the non-specific symptoms are systemic, they are consistent with metastatic cancer. However, a substantial proportion of early-stage lung, upper GI tract and haematological cancers were diagnosed, indicating that such symptoms may also provide an opportunity for timely diagnosis via a dedicated referral pathway such as the MDC, for these cancers at least. Furthermore, recent research indicates that a notable proportion of some solid tumour cancers presenting with non-specific symptoms were diagnosed at stages 1-3 (e.g. patients presenting with abdominal pain (as single symptom: 67\%, with other symptom(s): 62\%)), suggesting that early diagnosis for these patients remains a possibility. ${ }^{26}$

It is possible that a number of cancers, including those at early stage, were diagnosed 'incidentally', in that the symptom or symptoms leading to referral were not caused by the cancer.
However, this does not necessarily mean that the symptoms were not related to the cancer. ${ }^{27,28}$ They may, for example, have been of chronic non-malignant diseases sharing risk factors with the cancer. 29,30

There are some notable limitations. Firstly, this is a cohort study with no comparison group. This, in addition to the time-limited nature of the evaluation, has limited the ability to make judgements on the relative impact of the pathway being trialled and its long-term effect on patient outcomes. Whilst MDCs have provided an alternative rapid referral pathway for patients with suspected serious illness, further evaluative assessment should consider the balancing of benefit and harm regarding diagnostic investigations amongst this cohort, and for whom a cancer diagnosis occurs for a minority of patients. There was some missing data associated with the evaluation arrangements and, although measures were implemented to promote consistency regarding data collection and reporting, a certain degree of variation was evident due to localised data arrangements, something that warrants detailed consideration regarding the development of national data collection arrangements for similar pathways. Therefore, the study is dependent on the quality and completeness of the data collected. Finally, the study is based on a relatively small sample size that should be considered when assessing the pathway's key findings.

The ACE Programme has evaluated MDC-based pathways for the diagnosis of cancer amongst patients with non-specific symptoms. Additional analyses on MDC diagnostic testing activity and the results will elaborate on programme heterogeneity and associated learning, and will contribute to existing descriptive materials ${ }^{18}$ from the evaluation. Further analyses are also planned regarding the diagnosis of non-cancer conditions. Programme learning to date has informed the national development and implementation of rapid diagnostic centres (RDC) ${ }^{31}$ in England as part of the NHS Long Term Plan. ${ }^{32}$

It will be necessary to conduct further research on the longerterm impact of MDC pathways on outcomes for patients presenting with non-specific symptoms, and to build on existing work to assess patient experience within the MDC. ${ }^{33}$ The development of a viable comparator dataset will be essential to support future evaluative assessments, including a full evaluation of pathway health economics. These areas fall outside of the specific focus of this paper, but will provide valuable additional and complementary information in support of an emerging evidence base.

\section{CONCLUSIONS}

This evaluation of MDC pilots in England has demonstrated the potential of a dedicated referral pathway for patients presenting 
with non-specific but concerning symptoms, and provides a platform for the further development of the subject's evidence base. We found a strong association between a cancer diagnosis and patient age, and have identified GP 'clinical suspicion' as a strong predictor of cancer within this non-specific symptom cohort. This study has demonstrated that a cancer referral pathway for patients with non-specific but concerning symptoms could be a valuable addition to the referral options for suspected cancer, across a broad range of cancer sites.

\section{ACE MDC PROJECTS}

Airedale MDC pilot: Dr Alan Hart Thomas, Respiratory Consultant; Dawn Gulliford, Cancer Patient Services manager; Dr Helena Rolfe, Cancer Lead GP; Airedale MDC clinical team. Greater Manchester MDC pilots: Dr Matthias Hohmann, Oldham GP Cancer Lead; Chris Repperday, Data Analyst; Susan Sykes, Senior Programme Manager; Dr Sarah Taylor, Greater Manchester GP Cancer Lead; Greater Manchester MDC clinical teams. Leeds MDC pilot: Angie Craig, LTHT Assistant Director of Operations and Diagnostic Lead; James Dawson, Assistant Information Manager; Dr Sarah Forbes, GP Cancer Lead; Helen Ryan, Macmillan Leeds Cancer Programme Project Lead (Early Diagnosis); Dr Rob Turner, Consultant Clinical Oncologist. Leeds ACE MDC Clinical Team and Steering Group. London MDC pilots: Mush Ahmad, Data Manager; Donna Chung, Head of Centre for Cancer Outcomes, North Central and East London Cancer Alliance (formerly London Cancer, hosted by UCL Partners); Dr David Graham, Consultant Gastroenterologist; Dr Andrew Millar, Consultant Gastroenterologist; Sara Taiyari, Senior Project Manager; London MDC clinical teams. Oxford MDC pilot: $\mathrm{Dr}$ Claire Friedemann Smith, SCAN Researcher; Prof. Fergus Gleeson, Consultant Radiologist; Dr Shelley Hayles, Planned Care and Cancer Clinical Lead; Zoe Kaveney, Senior Project Manager; Dr Brian Nicholson, Macmillan GP and Senior Clinical Researcher; Oxford MDC clinical team.

\section{ACKNOWLEDGEMENTS}

We would like to thank the following: Sean Duffy (West Yorkshire and Harrogate Cancer Alliance), Sara Hiom (Cancer Research UK), Rosie Loftus (Macmillan Cancer Support) and Carol Ferguson (West Yorkshire and Harrogate Cancer Alliance) and the NAEDI Steering Group (https://www.cancerresearchuk.org/health-professional/ diagnosis/early-diagnosis-initiative) for forming and launching the MDC initiative; MDC patients; MDC project clinical teams from: Airedale General Hospital; Manchester University NHS Foundation Trust (Wythenshawe Hospital) and The Northern Care Alliance (Royal Oldham Hospital); Leeds St James University Hospital (Specialist Cancer Centre); London: North Middlesex University Hospital, University College London Hospital (Specialist Cancer Centre), Southend University Hospital and Queens (BHRUT) \& the Royal Free Hospital; Oxford University Hospitals Trust (Specialist Cancer Centre). Cancer Research Policy Unit; ACE Programme partners: NHS England, Cancer Research UK and Macmillan Cancer Support. Willie Hamilton is co-PI and Greg Rubin steering group chair of CanTest, a Cancer Research UK Catalyst Award [C8640/A23385].

\section{AUTHOR CONTRIBUTIONS}

D.C. wrote the paper and contributed to data interpretation. V.P. provided overall data management, contributed to statistical analysis and interpretation and supported the paper's development and review. K.F. contributed to data interpretation and supported the paper's development and review. S.W.D. and D.V. provided statistical analyses and interpretation and contributed to the paper's development and review. G.R. and W.H. contributed to the clinical interpretation of the study and supported the paper's development and review. The five MDC project teams and clinical staff developed and managed the operational pilot sites and generated data for the study. MDC clinicians also supported the interpretation of MDC data.

\section{ADDITIONAL INFORMATION}

Ethics approval and consent to participate The ACE evaluation was classified as a service evaluation and was therefore not subject to ethics approval.
Consent to publish Not applicable.

Data availability Pseudoanonymised data were supplied by the Multidisciplinary Diagnostic Centres (MDC) - ACE (Cancer Research UK) is the custodian of the data on behalf of the MDCs and for the duration of the analysis-the data are not publicly available at present.

Competing interests The authors declare no competing interests.

Funding information The ACE Programme (MDC) was a joint early diagnosis of cancer initiative between NHS England, Cancer Research UK and Macmillan Cancer Support. Funding for the Programme Management of the ACE Programme was provided by Cancer Research UK. S.W.D., D.V., W.H. and G.R. participated in this work as part of the responsive programme of the Policy Research Unit in Cancer Awareness, Screening and Early Diagnosis, which is funded by the Department of Health's Policy Research Programme. The five MDC projects received programme funding to support the development and trial of pilot pathways in accordance with their status as ACE Programme sites.

Supplementary information is available for this paper at https://doi.org/10.1038/ s41416-020-0947-y.

Publisher's note Springer Nature remains neutral with regard to jurisdictional claims in published maps and institutional affiliations.

\section{REFERENCES}

1. Neal, R., Din, N., Hamilton, W., Ukoumunne, O., Carter, B., Stapley, S. et al. Comparison of cancer diagnostic intervals before and after implementation of NICE guidelines: analysis of data from the UK General Practice Research Database. Br. J. Cancer 110, 584 (2014).

2. National Institute for Health and Care Excellence. Suspected cancer: recognition and referral. NICE guideline (NG12, 2015).

3. Koo, M. M., Hamilton, W., Walter, F. M., Rubin, G. P. \& Lyratzopoulos, G. Symptom Signatures and Diagnostic Timeliness in Cancer Patients: A Review of Current Evidence. Neoplasia, Feb 20, 165-174 (2018).

4. Jørgensen, S. F., Ravn, P., Thorsen, S. \& Worm, S. W. Characteristics and outcomes in patients with non-specific symptoms and signs of cancer referred to a fast track cancer patient pathway; a retrospective cohort study. BMC Cancer 17, 809 (2017)

5. Nicholson, B., Hamilton, W., O'Sullivan, J., Aveyard, P. \& Hobbs, R. Weight loss as a predictor of cancer in primary care: a systematic review and meta-analysis. Br. J. Gen. Pract. 68, e311-e322 (2018).

6. Pearson, C., Poirier, V., Fitzgerald, K., Rubin, G. \& Hamilton, W. Cross-sectional study using primary care and cancer registration data to investigate patients with cancer presenting with non-specific symptoms. BMJ Open 10, e033008 (2020).

7. Lyratzopoulos, G., Wardle, J. \& Rubin, G. Rethinking diagnostic delay in cancer: how difficult is the diagnosis? BMJ 349, g7400 (2014).

8. Lyratzopoulos, G., Neal, R. D., Barbiere, J. M., Rubin, G. P. \& Abel, G. A. Variation in number of general practitioner consultations before hospital referral for cancer: findings from the 2010 National Cancer Patient Experience Survey in England. Lancet Oncol. 13, 353-365 (2012).

9. Renzi, C., Lyratzopoulos, G., Card, T., Chu, T. P. C., Macleod, U. \& Rachet, B. Do colorectal cancer patients diagnosed as an emergency differ from nonemergency patients in their consultation patterns and symptoms? A longitudinal data-linkage study in England. Br. J Cancer 115, 866-875 (2016).

10. Richards, M. A. The size of the prize for earlier diagnosis of cancer in England. $B r$. J. Cancer 101, S125-S129 (2009).

11. Nicholson, B. D., Oke, J., Friedemann Smith, C., Philips, J. A., Lee, J., Abel, L. et al. The Suspected CANcer (SCAN) pathway: protocol -for evaluating a new standard of care for patients with non-specific symptoms of cancer. BMJ Open 8, e018168 (2018).

12. McPhail, S., Elliss-Brookes, L., Shelton, J., Ives, A., Greenslade, M., Vernon, S. et al. Emergency presentation of cancer and short-term mortality. Br. J. Cancer 109, 2027 (2013).

13. Mendonca, S. C., Abel, G. A., Saunders, C. L., Wardle, J. \& Lyratzopoulos, G. Prereferral general practitioner consultations and subsequent experience of cancer care: evidence from the English Cancer Patient Experience Survey. Eur. J. Cancer Care (Engl.) 25, 478-490 (2016).

14. Vedsted, P. \& Olesen, F. A differentiated approach to referrals from general practice to support early cancer diagnosis-the Danish three-legged strategy. $B r$. J. Cancer 112, S65-S69 (2015). 
15. Ingeman, M. L., Christensen, M. B., Bro, F., Knudsen, S. T. \& Vedsted, P. The Danish cancer pathway for patients with serious non-specific symptoms and signs of cancer-a cross-sectional study of patient characteristics and cancer probability. BMC Cancer 15, 421 (2015).

16. Moseholm, E. \& Lindhardt, B. Ø. Patient characteristics and cancer prevalence in the Danish cancer patient pathway for patients with serious non-specific symptoms and signs of cancer-A nationwide, population-based cohort study. Cancer Epidemiol. 50, 166-172 (2017).

17. Fuller, E., Fitzgerald, K. \& Hiom, S. Accelerate, coordinate, evaluate programme: a new approach to cancer diagnosis. Br. J. Gen. Pr. 66, 176-177 (2016).

18. ACE Programme (Cancer Research UK). Identifying distinguishing features of the MDC model within the five ACE projects. ACE Programme 2019. https://www. cancerresearchuk.org/sites/default/files/

identifying_distinguishing_features_of_the_mdc_model_within_the_five_mdc_projects_-_final_june_19.pdf (2019).

19. National Cancer Registration and Analysis Service, Public Health England. Cancer Outcome Cancer Outcomes and Services Dataset - version 8. http://www.ncin. org.uk/collecting_and_using_data/data_collection/cosd (2019).

20. Oken, M. M., Creech, R. H., Tormey, D. C., Horton, J., Davis, T. E., McFadden, E. T. et al. Toxicity and response criteria of the Eastern Cooperative Oncology Group. Am. J. Clin. Oncol. 5, 649e655 (1982).

21. NHS. https://www.datadictionary.nhs.uk/data_dictionary/nhs_business_definitions/ a/adult_comorbidity_evaluation_-_27_de.asp?shownav=1 (2019).

22. Hjertholm, P., Moth, G., Ingeman, M. L. \& Vedsted, P. Predictive values of GPs' suspicion of serious disease: a population-based follow-up study. Br. J. Gen. Pract. 64, e346-e53 (2014).

23. Søgaard, M., Thomsen, R. W., Bossen, K. S., Sørensen, H. T. \& Nørgaard, M. The impact of comorbidity on cancer survival: a review. Clin. Epidemiol. 5, 3-29 (2013). Published 2013 Nov. 1.

24. Grose, D., Devereux, G., Brown, L., Jones, R., Sharma, D., Selby, C. et al. Variation in comorbidity and clinical management in patients newly diagnosed with lung cancer in four Scottish centres. J. Thorac. Oncol. 6, 500-509 (2011).

25. Mills, K., Emery, J., Cheung, C., Hall, N., Birt, L. \& Walter, F. M. A qualitative exploration of the use of calendar landmarking instruments in cancer symptom research. BMC Fam. Pr. 15, 167 (2014).

26. Koo, M. M, Swann, R., McPhail, S., Abel, G. A, Elliss-Brookes, L., Rubin, G. et al. Presenting symptoms of cancer and stage at diagnosis: evidence from a cross-sectional, population-based study. Lancet Oncol. https://doi.org/10.1016/ S1470-2045(19)30595-9 (2019).

27. Ades, A. E., Biswas, M., Welton, N. J. \& Hamilton, W. Symptom lead time distribution in lung cancer: natural history and prospects for early diagnosis. Int. J. Epidemiol. 43, 1865-1873 (2014).

28. Biswas, M., Ades, A. E. \& Hamilton, W. Symptom lead times in lung and colorectal cancers: what are the benefits of symptom-based approaches to early diagnosis? Br. J. Cancer 112, 271-277 (2015).

29. Kenzik, K. M., Kent, E. E., Martin, M. Y., Bhatia, S. \& Pisu, M. Chronic condition clusters and functional impairment in older cancer survivors: a population-based study. J. Cancer Surviv 10, 1096-1103 (2016).

30. Ohwaki, K., Fumiyasu, E., Masaki, S. \& Akiko, F. Comorbidities as predictors of incidental prostate cancer after Holmium laser enucleation of the prostate: diabetes and high-risk cancer. Aging Male 20, 257-260 (2017).

31. NHS England. https://www.england.nhs.uk/wp-content/uploads/2019/07/rdcvision-and-1920-implementation-specification.pdf (2019).

32. NHS England. https://www.england.nhs.uk/long-term-plan/ (2019).

33. Howse, J. \& Rubin, G. ACE Wave 2 MDC Patient Experience Survey. ACE Programme. https://www.cancerresearchuk.org/health-professional/diagnosis/ accelerate-coordinate-evaluate-ace-programme/multidisciplinary-diagnosticcentres-mdcs\#info_gallery_3 (2019).

(i) Open Access This article is licensed under a Creative Commons Attribution 4.0 International License, which permits use, sharing, adaptation, distribution and reproduction in any medium or format, as long as you give appropriate credit to the original author(s) and the source, provide a link to the Creative Commons license, and indicate if changes were made. The images or other third party material in this article are included in the article's Creative Commons license, unless indicated otherwise in a credit line to the material. If material is not included in the article's Creative Commons license and your intended use is not permitted by statutory regulation or exceeds the permitted use, you will need to obtain permission directly from the copyright holder. To view a copy of this license, visit http://creativecommons. org/licenses/by/4.0/.

(c) The Author(s) 2020 